\title{
THE BEREZIN SYMBOL AND MULTIPLIERS OF FUNCTIONAL HILBERT SPACES
}

\author{
SEMRA KILIC \\ (Communicated by Palle E. T. Jorgensen)
}

\begin{abstract}
This paper focuses on a multiplicative property of the Berezin symbol $\tilde{A}$, of a given linear map $A: \mathscr{H} \mapsto \mathscr{H}$, where $\mathscr{H}$ is a functional Hilbert space of analytic functions. We show $\widetilde{A B}=\widetilde{A} \widetilde{B}$ for all $B$ in $\mathscr{B}(\mathscr{H})$ if and only if $A$ is a multiplication operator $M_{\varphi}$, where $\varphi$ is a multiplier. We also present a version of this result for vector-valued functional Hilbert spaces.
\end{abstract}

\section{INTRODUCTION}

Let $n$ be a fixed positive integer and let $\Omega$ be a region in $\mathbf{C}^{n}$. A functional Hilbert space $\mathscr{H}$ is a Hilbert space of analytic functions on $\Omega$ such that the point evaluations are bounded, linear functionals. By the Riesz-representation theorem there exists, for each $z$ in $\Omega$, a unique element $K_{z}$ of $\mathscr{H}$ such that $f(z)=\left\langle f, K_{z}\right\rangle$ for all $f$ in $\mathscr{H}$. The function $K$ on $\Omega \times \Omega$, defined by $K(z, w)=K_{w}(z)$, is called the reproducing kernel function of $\mathscr{H}$. Let $k_{z}=\frac{K_{i}}{\left\|K_{z}\right\|}$ be the normalized reproducing kernel function. For a given linear map $A: \mathscr{H} \mapsto \mathscr{H}$, the Berezin symbol $\tilde{A}$ (see [1]) of a map $A$ of $\mathscr{H}$ into itself is defined by

$$
\tilde{A}(z)=\left\langle A k_{z}, k_{z}\right\rangle \text {. }
$$

It is known that the map $A \mapsto \tilde{A}$ is injective (see [3]). A function $\varphi$ defined on $\Omega$ is a multiplier of $\mathscr{H}$ if $\varphi \cdot f$ is in $\mathscr{H}$, for all $f$ in $\mathscr{H}$. Let $\mathscr{B}(\mathscr{H})$ denote the set of all bounded, linear operators from $\mathscr{H}$ into $\mathscr{H}$. The multiplication operator $M_{\varphi}: \mathscr{H} \mapsto \mathscr{H}$ defined by $M_{\varphi} f=\varphi \cdot f$ is in $\mathscr{B}(\mathscr{H})$, when $\varphi$ is a multiplier of $\mathscr{H}$.

\section{THE MULTIPLICATIVE PROPERTY OF THE BEREZIN SYMBOL ON A FUNCTIONAL HILBERT SPACE}

Theorem 1. Let $A$ be a bounded operator on $\mathscr{H}$. Then

$$
\widetilde{A B}(z)=\widetilde{A}(z) \widetilde{B}(z)
$$

Received by the editors November 22, 1993 and, in revised form, March 18, 1994 and May 12, 1994; the contents of this paper were presented to the AMS at a Special Session of the meeting in Dayton, Ohio, October 1992.

1991 Mathematics Subject Classification. Primary 46E22; Secondary 47B35, 47B38.

Key words and phrases. Berezin symbol, multiplier, functional Hilbert spaces, multiplication operators, Toeplitz operators. 
for all $B$ in $\mathscr{B}(\mathscr{H})$ if and only if $A$ is a multiplication operator, $M_{\varphi}$, where $\varphi$ is a multiplier. Moreover, $\varphi=\widetilde{A}$.

Before proceeding with the proof, we need the following:

Lemma 1. When $\varphi$ is a multiplier of $\mathscr{H}, \widetilde{M}_{\varphi}(z)=\varphi(z)$.

Proof. $\widetilde{M}_{\varphi}(z)=\left\langle M_{\varphi} k_{z}, k_{z}\right\rangle=\left\langle\varphi k_{z}, k_{z}\right\rangle=\varphi(z)$.

Lemma 2. The Berezin symbol of $f \otimes g$, for $f, g$ in $\mathscr{H}$, is

$$
(\widetilde{f \otimes g})(z)=\frac{\overline{g(z)}}{\left\|K_{z}\right\|^{2}} f(z), \quad z \in \Omega .
$$

Proof. For $f$ and $g$ in $\mathscr{H}$ and $z$ in $\Omega$,

$$
\begin{aligned}
(\widetilde{f \otimes g})(z) & =\left\langle(f \otimes g) \frac{K_{z}}{\left\|K_{z}\right\|} \frac{K_{z}}{\left\|K_{z}\right\|}\right\rangle \\
& =\frac{1}{\left\|K_{z}\right\|^{2}}\left\langle K_{z}, g\right\rangle\left\langle f, K_{z}\right\rangle .
\end{aligned}
$$

By the reproducing property of the kernel function, we have

$$
(\widetilde{f \otimes g})(z)=\frac{\overline{g(z)}}{\left\|K_{z}\right\|^{2}} f(z), \quad f, q \in \mathscr{H} .
$$

Proof of Theorem 1. Suppose $\widetilde{A B}(z)=\widetilde{A}(z) \widetilde{B}(z)$ for all $B$ in $\mathscr{B}(\mathscr{H})$. Let $B=f \otimes g$ for $f$ and $g$ in $\mathscr{H}$. Then, by Lemma 2,

$$
\widetilde{A B}(z)=(A \widetilde{f \otimes g})(z)=\frac{\overline{g(z)}}{\left\|K_{z}\right\|^{2}}(A f)(z) .
$$

By the hypothesis, we have

$$
\frac{\overline{g(z)}}{\left\|K_{z}\right\|^{2}}(A f)(z)=\frac{\overline{g(z)}}{\left\|K_{z}\right\|^{2}} \widetilde{A}(z) f(z),
$$

which reduces to

$$
(A f)(z)=\widetilde{A}(z) f(z)
$$

for all $f$ in $\mathscr{H}$. Hence $A=M_{\tilde{A}}$.

Conversely, if $A$ is a multiplication operator, $M_{\varphi}$, where $\varphi$ is a multiplier,

$$
\widetilde{M_{\varphi} B}=\left\langle M_{\varphi} B k_{z}, k_{z}\right\rangle=\varphi(z) \frac{\left(B k_{z}\right)(z)}{\left\|K_{z}\right\|}
$$

for all $B$ in $\mathscr{B}(\mathscr{H})$. By Lemma 1, we have

$$
\widetilde{M_{\varphi} B}(z)=\widetilde{M}_{\varphi}(z) \widetilde{B}(z)
$$

for all $B$ in $\mathscr{B}(\mathscr{H})$.

Corollary 1. Let $B$ be in $\mathscr{B}(\mathscr{H})$. Then

$$
\widetilde{A B}(z)=\widetilde{A}(z) \widetilde{B}(z)
$$

for all $A$ in $\mathscr{B}(\mathscr{H})$ if and only if $B=M_{\psi}^{*}$, where $\psi$ is a multiplier.

Proof. The assertion follows from Theorem 1 and the fact that $\widetilde{T^{*}}(z)=\overline{\widetilde{T}}(z)$, for all $T$ in $\mathscr{B}(\mathscr{H})$. 
The Hardy space $H^{2}$ consists of the complex-valued analytic functions on the unit disk $\mathbf{D}$ such that the Taylor coefficients are square summable. A calculation shows that $K_{z}=\frac{1}{1-\bar{z} w}$ has the reproducing property (see [4]). Let $P$ denote the orthogonal projection of $L^{2}(\partial \mathbf{D})$ onto $H^{2}$, and let $\varphi$ be a bounded measurable function. Then the Toeplitz operator, $T_{\varphi}$, induced by $\varphi$ is defined by $T_{\varphi} f=$ $P(\varphi f)$, for all $f$ in $H^{2}$.

Corollary 2. Let $A$ be a bounded operator on $H^{2}$. Then

$$
\widetilde{A B}(z)=\widetilde{A}(z) \widetilde{B}(z)
$$

for all $B$ in $\mathscr{B}\left(H^{2}\right)$ if and only if $A$ is a Toeplitz operator, $T_{\varphi}$, induced by $\varphi$ in $H^{\infty}$. Moreover $\varphi=\tilde{A}$.

Proof. The multiplication operators on $H^{2}$ are the analytic Toeplitz operators.

We should mention that Corollary 2 is also true if one replaces $H^{2}$ by the Bergman space or any of the weighted Bergman spaces. (For analytic Toeplitz operators on weighted Bergman spaces see [6].)

\section{THE MULTIPLICATIVE PROPERTY OF THE BEREZIN SYMBOL} ON THE ANALYTIC REPRODUCING KERNEL SPACE, $\mathscr{H}=\mathscr{H}_{0} \otimes \mathscr{C}$

Let $\mathscr{K}_{0}$ be a functional Hilbert space of (scalar-valued) analytic functions on $\Omega$ with the reproducing kernel function $K_{z}$, for each fixed $z$ in $\Omega$. Let $\mathscr{C}$ be a separable Hilbert space, and let $\mathscr{H}$ be the functional Hilbert space of $\mathscr{C}$-valued functions, $\mathscr{H}=\mathscr{H}_{0} \otimes \mathscr{C}$. The reproducing kernel function of $\mathscr{H}$, $\mathfrak{I}_{z}: \mathscr{C} \mapsto \mathscr{C}$, is defined by $\mathfrak{I}_{z}(u)=K_{z} \otimes u$, where $u$ is in $\mathscr{C}$.

The evaluation functional $E_{z}: \mathscr{H} \mapsto \mathscr{C}$, defined by $E_{z} f=f(z)$, for $z$ in $\Omega$, is bounded (see [2], Lemma 3.2). For $f \in \mathscr{H}, u$ in $\mathscr{C}$, we have

$$
\left\langle f, E_{z}^{*} u\right\rangle_{\mathscr{L}}=\langle f(z), u\rangle_{\mathscr{C}} .
$$

We also have the reproducing property of the kernel function, that is

$$
\left\langle f, \mathfrak{I}_{z}(u)\right\rangle_{\mathscr{E}}=\langle f(z), u\rangle_{\mathscr{C}} .
$$

Therefore, $E_{z}^{*} u=\mathfrak{I}_{z}(u)$, for all $u$ in $\mathscr{C}$. By the reproducing property of the kernel function, we have $\left\|\mathfrak{I}_{z}(u)\right\|^{2}=K_{z}(z)\|u\|^{2}$, where $u$ is in $\mathscr{C}$, and hence $\left\|\mathfrak{I}_{z}\right\|=\sqrt{K_{z}(z)}=\left\|E_{z}\right\|$.

Let $\mathscr{K}_{z}=\frac{\mathfrak{J}_{-}}{\left\|\mathcal{J}_{-}\right\|}$be the normalized reproducing kernel function, and let $A$ be a bounded linear operator on $\mathscr{H}$. Then the Berezin symbol $\tilde{A}$ of $A$ is defined by

$$
\widetilde{A}(z)=\mathscr{K}_{z}^{*} A \mathscr{K}_{z} .
$$

Lemma 3. An operator $A$ is a multiplication operator if and only if, for each fixed $z$ in $\Omega, A^{*} E_{z}^{*}=E_{z}^{*} \Phi(z)^{*}$ for some operator $\Phi(z)$ in $\mathscr{B}(\mathscr{C})$. Moreover, in this case, $A$ is the operator of multiplication by the function $z \mapsto \Phi(z)$.

Proof. Let $z$ be fixed in $\Omega$. Suppose $A$ is a multiplication operator, $M_{\Phi}$, induced by $\Phi: \Omega \rightarrow \mathscr{B}(\mathscr{C})$. We observe that

$$
E_{z} M_{\Phi} f=M_{\Phi} f(z)=\Phi(z) f(z)=\Phi(z) E_{z} f \text { for all } f \text { in } \mathscr{H} .
$$

Then we have $E_{z} M_{\Phi}=\Phi(z) E_{z}$, for some operator $\Phi(z)$ in $\mathscr{B}(\mathscr{C})$. 
Conversely, let $A$ be a bounded operator on $\mathscr{H}$ such that $A^{*} E_{z}^{*}=E_{z}^{*} \Phi(z)^{*}$ for some operator $\Phi(z)$ in $\mathscr{B}(\mathscr{C})$. For $u$ in $\mathscr{C}$, we have

$$
\left\langle f, A^{*} E_{z}^{*} u\right\rangle_{\mathscr{H}}=\left\langle A f, E_{z}^{*} u\right\rangle_{\mathscr{H}}=\langle(A f)(z), u\rangle_{\mathscr{C}} \text { for all } f \text { in } \mathscr{H} \text {. }
$$

On the other hand, for $u$ in $\mathscr{C}$, we have $\left\langle f, E_{z}^{*} \Phi(z)^{*} u\right\rangle=\langle\Phi(z) f(z), u\rangle$, for all $f$ in $\mathscr{H}$. Then $\langle(A f)(z), u\rangle=\langle\Phi(z) f(z), u\rangle$, for all $f$ in $\mathscr{H}$ and $u$ in $\mathscr{C}$. Therefore, $(A f)(z)=\Phi(z) f(z)$, for all $f$ in $\mathscr{H}$.

Theorem 2. Let $A$ be a bounded operator on $\mathscr{H}$. Then

$$
\widetilde{A B}(z)=\widetilde{A}(z) \widetilde{B}(z)
$$

for all $B$ in $\mathscr{B}(\mathscr{H})$ if and only if $A=M_{\Phi}$, where $\Phi: \Omega \mapsto \mathscr{B}(\mathscr{C})$.

Proof. We observe that $E_{z} M_{\Phi} f=\Phi(z) f(z)$, for all $f$ in $\mathscr{H}$. Then $E_{z} M_{\Phi} E_{z}^{*}$ $=\Phi(z) E_{z} E_{z}^{*}$ and $E_{z} M_{\Phi} B E_{z}^{*}=\Phi(z) E_{z} B E_{z}^{*}$, for all $B$ in $\mathscr{B}(\mathscr{H})$. Since $E_{z} E_{z}^{*}=K_{z}(z) I_{\mathscr{C}}$, we have $\widetilde{M}_{\Phi}=\Phi(z)$ and

$$
\widetilde{M_{\Phi}} B(z)=\Phi(z) \frac{E_{z} B E_{z}^{*}}{\left\|I_{z}\right\|^{2}}=\widetilde{M}_{\Phi}(z) \widetilde{B}(z) \text { for all } B \text { in } \mathscr{B}(\mathscr{H}) \text {. }
$$

Conversely, suppose that $A$ is a bounded operator such that $\widetilde{A B}(z)=$ $\widetilde{A}(z) \widetilde{B}(z)$ for all $B$ in $\mathscr{B}(\mathscr{H})$. Then from the definitions, we get

$$
E_{z} A B E_{z}^{*}=\frac{1}{\left\|E_{z}\right\|^{2}} E_{z} A E_{z}^{*} E_{z} B E_{z}^{*} \text { for all } B \text { in } \mathscr{B}(\mathscr{H}) \text {. }
$$

For $u$ and $v$ in $\mathscr{C}$, we have

$$
\left\langle E_{z} A B E_{z}^{*} u, v\right\rangle=\left\langle\frac{E_{z} A E_{z}^{*}}{\left\|E_{z}\right\|^{2}} E_{z} B E_{z}^{*} u, v\right\rangle=\left\langle\tilde{A}(z) E_{z} B E_{z}^{*} u, v\right\rangle .
$$

Then we have

$$
\left\langle B E_{z}^{*} u, A^{*} E_{z}^{*} v\right\rangle=\left\langle B E_{z}^{*} u, E_{z}^{*} \tilde{A}(z)^{*} v\right\rangle .
$$

For each fixed nonzero $u, B E_{z}^{*} u$ runs through all vectors in $\mathscr{C}$ as $B$ runs through all elements of $\mathscr{B}(\mathscr{H})$. Thus we see that $A^{*} E_{z}^{*}=E_{z}^{*} \widetilde{A}(z)^{*}$, for all $z$ in $\Omega$. Therefore $A$ is a multiplication operator, $M_{\widetilde{A}}$, by Lemma 3 .

Let us note that if we take $\mathscr{C}$ to be $\mathrm{C}$ and define $\mathscr{K}_{z}=k_{z} \otimes 1$, the sufficiency proof of Theorem 2 will also work for Theorem 1, the scalar-valued case.

Let $\mathbf{N}=\{0,1,2, \ldots\}$ denote the set of nonnegative integers. The set $\mathbf{N}^{n}$ is partially ordered by setting $\mathbf{I}=\left(i_{1}, i_{2}, \ldots, i_{n}\right) \geq\left(j_{1}, j_{2}, \ldots, j_{n}\right)=\mathbf{J}$ if and only if $i_{k} \geq j_{k}$ for $k=1,2, \ldots, n$. If $z=\left(z_{1}, z_{2}, \ldots, z_{n}\right)$ is in $\Omega$, then we set $z^{I}=z_{1}^{i_{1}} \cdot z_{2}^{i_{2}} \cdots \cdots z_{n}^{i_{n}}$. We denote by $H^{2}(n) \otimes \mathscr{C}$, where $H^{2}(n)=H^{2} \otimes H^{2} \otimes \cdots \otimes H^{2}$ ( $n$ copies), the set of all vector-valued analytic functions $f: \mathbf{D}^{n} \mapsto \mathscr{C}$ with power series expansion $f(z)=\sum_{I \in \mathbf{N}^{n}} z^{I} v_{I}$, with $v_{I}$ in $\mathscr{C}$ and $z$ in $\mathbf{D}^{n}$, such that $\sum_{I \in \mathbf{N}^{n}}\left\|v_{I}\right\|_{\mathscr{C}}^{2}<\infty$.

The space $H^{2}(n) \otimes \mathscr{C}$ is a Hilbert space with the reproducing kernel function, $\mathfrak{I}_{z}: \mathscr{C} \mapsto H^{2}(n) \otimes \mathscr{C}$, for $z$ in $\mathbf{D}^{n}$, defined by $\mathfrak{I}_{z}(u)=K_{z} \otimes u$, where $u$ is in $\mathscr{C}$ and $K_{z}(w)=\sum_{I \in \mathrm{N}^{n}} \bar{z}^{I} w^{I}$ is the reproducing kernel function for $H^{2}(n)$ (see [5]). Let $H^{\infty}(n)(\mathscr{B}(\mathscr{C}))$ denote the Banach space of all bounded analytic functions $\Phi: \mathbf{D}^{n} \mapsto \mathscr{B}(\mathscr{C})$ with the norm $\|\Phi\|_{\infty}=\sup \left\{\|\Phi(z)\|\right.$, for $\left.z \in \mathbf{D}^{n}\right\}$. 
For every $\Phi$ in $H^{\infty}(n)(\mathscr{B}(\mathscr{C}))$, we can define the analytic Toeplitz operator $T_{\Phi}$ in $\mathscr{B}\left(H^{2}(n) \otimes \mathscr{C}\right)$ as follows:

$$
\left(T_{\Phi} f\right)(z)=\Phi(z) f(z), \quad z \text { in } \mathbf{D}^{n}, f \text { in } H^{2}(n) \otimes \mathscr{C} .
$$

For the boundedness of the map $T_{\Phi}$ see [2].

Corollary 3. Let $A$ be a bounded operator on $H^{2}(n) \otimes \mathscr{C}$. Then

$$
\widetilde{A B}(z)=\widetilde{A}(z) \widetilde{B}(z)
$$

for all $B$ in $\mathscr{B}\left(H^{2}(n) \otimes \mathscr{C}\right)$ if and only if $A=T_{\Phi}$, where $\Phi$ is in $H^{\infty}(n)(\mathscr{B}(\mathscr{C}))$.

\section{ACKNOWLEDGMENT}

I thank my thesis advisor, Eric Nordgren, for his encouragement and support throughout my work. Also, I thank the referee for several helpful suggestions.

\section{REFERENCES}

1. F. A. Berezin, Covariant and contravariant symbols for operators, Math. USSR-Izv. 6 (1972), $1117-1151$.

2. R. E. Curto and N. Salinas, Generalized Bergman kernels and the Cowen-Douglas theory, Amer. J. Math. 106 (1984), 447-488.

3. V. Guillemin, Toeplitz operators in n-dimensions, Integral Equations Operator Theory 7 (1984), 145-204.

4. P. R. Halmos, A Hilbert space problem book, Van Nostrand, Princeton, NJ, 1967.

5. G. T. Adams, J. Froelich, and V. I. Paulsen, Analytic reproducing kernels and factorization, Indiana Univ. Math. J. 43 (1994), 839-856.

6. K. Zhu, Operator theory in function spaces, Marcel Dekker, New York, 1990.

Department of Mathematics, University of New Hampshire, Durham, New Hampshire 03824

Current address: Simon's Rock College of Bard, 84 Alford Rd., Great Barrington, Massachusetts 01230-9702 